\title{
Optical Biopsy Mapping for Minimally Invasive Cancer Screening
}

\author{
Peter Mountney $^{1,2}$, Stamatia Giannarou ${ }^{2}$, Daniel Elson ${ }^{2}$, \\ and Guang-Zhong Yang ${ }^{1,2}$ \\ ${ }^{1}$ Department of Computing \\ ${ }^{2}$ Institute of Biomedical Engineering Imperial College, London SW7 2BZ, UK \\ \{peter.mountney, stamatia.giannarou03, ds.elson, \\ g.z.yang\} @imperial.ac.uk
}

\begin{abstract}
The quest for providing tissue characterization and functional mapping during minimally invasive surgery (MIS) has motivated the development of new surgical tools that extend the current functional capabilities of MIS. Miniaturized optical probes can be inserted into the instrument channel of standard endoscopes to reveal tissue cellular and subcellular microstructures, allowing excision-free optical biopsy. One of the limitations of such a point based imaging and tissue characterization technique is the difficulty of tracking probed sites in vivo. This prohibits large area surveillance and integrated functional mapping. The purpose of this paper is to present an image-based tracking framework by combining a semi model-based instrument tracking method with vision-based simultaneous localization and mapping. This allows the mapping of all spatio-temporally tracked biopsy sites, which can then be re-projected back onto the endoscopic video to provide a live augmented view in vivo, thus facilitating re-targeting and serial examination of potential lesions. The proposed method has been validated on phantom data with known ground truth and the accuracy derived demonstrates the strength and clinical value of the technique. The method facilitates a move from the current point based optical biopsy towards large area multi-scale image integration in a routine clinical environment.
\end{abstract}

\section{Introduction}

With recent advances in biophotonics and surgical instrumentation, there is an increasing demand to bring cellular and molecular imaging modalities to an in vivo, in situ setting to allow for real-time tissue characterization, functional assessment and intra-operative guidance. Miniaturization of the confocal laser scanning microscope, for example, has led to imaging probes that can be inserted into the instrument channel of a standard endoscope to visualize cellular and subcellular microstructures to provide 'optical biopsy' without excision of tissue. Following the application of a contrast agent, this can allow for the detection of colorectal adenomas, disruption in the pit pattern of the colon, angiogenesis, and neoplasia in Barrett's esophagus [1]. It has also been used without a contrast agent to detect malignant disruption of the bronchial basement membrane using elastin autofluorescence [2]. Other techniques that enable microscopic detection and characterization of tissue include Optical Coherence 
Tomography (OCT), two photon excited fluorescence and high magnification endoscopy [3]. There have also been successful clinical trials of techniques that acquire detailed spectroscopic information for cancer detection, for example using the timeor wavelength-resolved fluorescence or Raman properties.

For in vivo applications, all of these techniques suffer from the limitation of only providing a small, localized probe region whilst the organs of interest may require a large surface area to be surveyed. Technically, the main difficulty of tracking the optical biopsy sites is that these probes leave no marks on the tissue. Furthermore, the optical biopsy sites move in and out of the view in a standard endoscope image as the examination progresses and may deform as a result of respiration or tissueinstrument interaction. Current approaches to long-term tissue-instrument tracking assume the use of rigid laparoscopes and availability of optical markers [4]. Structure from motion has been used to reconstruct 3D tissue models, but it suffers from drift and does not work well when revisiting biopsy sites [5]. For extending the effective field-of-view of the endoscopic image, image mosaicing [6] and dynamic view expansion [7] have been used to reconstruct enlarged field-of-views, although these techniques tend not to explicitly deal with motion parallax.

In practice, optical probes are typically introduced through the instrument channel while holding the endoscope stationary. Since the probe needs to be placed in contact with the tissue when the optical biopsy takes place, tracking the tip of the probe enables the localization of the biopsy site. To this end, it is necessary to take into account scale, rotation and illumination changes when tracking the tool. Current approaches to needle and surgical instrument tracking may be applicable [8,9], but a combined approach by integrating probe tracking with a 3D probabilistic map built in situ using only white light endoscopic images with no additional fiducials can ensure robustness and practical clinical use. This work proposes an image-based tracking system based on SLAM (Simultaneous Localization and Mapping) for optical probes. This will allow for subsequent localization and contextual analysis of microstructures or guiding real tissue biopsy. The main contribution of this paper is to combine SLAM with probe tracking to create a 3D model of the tissue surface and spatiotemporally tracked optical biopsy sites. These biopsy sites are subsequently reprojected back onto the image plane to provide a live augmented view in vivo, thus facilitating re-targeting and serial examination. The proposed method has been
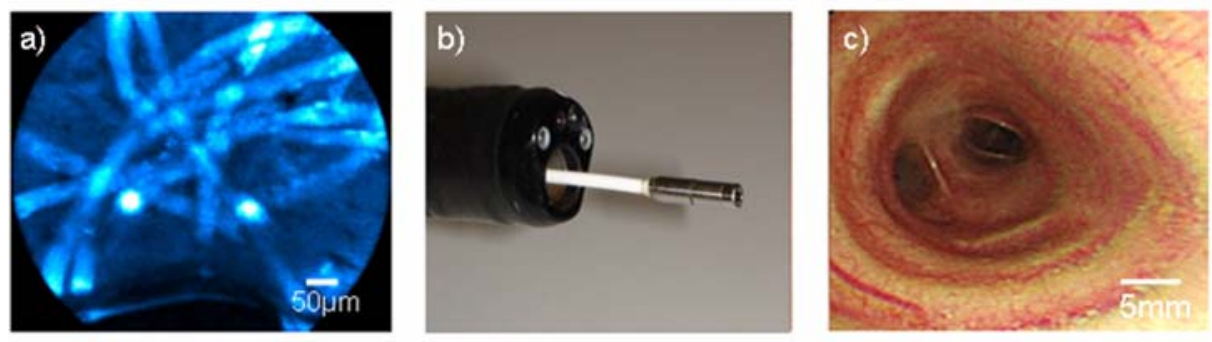

Fig. 1. (a) A typical microconfocal fluorescence image showing the microstructure of a sample, (b) the relative configuration of a confocal fluorescence probe when inserted through the instrument channel of a standard endoscope, and (c) a typical endoscopic white light image of the bronchus used for navigation 
validated on phantom data with known ground truth. The method will facilitate a shift from the current point based optical biopsy towards multi-scale image integration in a routine clinical environment.

\section{Methods}

\subsection{Probabilistic Mapping}

The first step of the proposed tracking framework is to establish a probabilistic mapping of the environment. Previous work on SLAM based approaches has shown the ability to generate 3D tissue models and recover the relative pose of the endoscope [10]. A long-term map is generated making it more resilient to drift and error accumulation over time, and thus is well suited to returning to previously targeted areas. In this work, a vision based sequential approach has been used. This is based on an Extended Kalman Filter (EKF) framework with state vector $x$ containing the position $\left(c_{x}, c_{y}, c_{z}\right)$, orientation $\left(c_{q 1}, c_{q 2}, c_{q 3}, c_{q 4}\right)$, translational velocity $\left(v_{x}, v_{y}, v_{z}\right)$ and angular velocity $\left(\omega_{x}, \omega_{y}, \omega_{z}\right)$ of the endoscope. In addition, the state vector also stores $3 \mathrm{D}$ locations of salient features in the map $\left(y_{x}, y_{y}, y_{z}\right)$. A constant velocity, constant angular velocity motion model is used to predict the endoscope's motion with Gaussian noise. Accompanying the state vector is the covariance matrix which stores the uncertainty of the endoscope and feature locations in 3D. In this sequential map building approach, new features are added to the map on the fly by feature matching constrained by epipolar geometry to estimate their $3 \mathrm{D}$ positions relative to the endoscopic camera.

\subsection{Biopsy Site Estimation}

The initial position of the biopsy site in the image plane is estimated through probe tracking. In this work, no marker was attached and no changes were made to the colour of the imaging probe. The technique exploits the fact that the camera is relatively static when the biopsy is taken. The segmentation of the tool is achieved by combining background subtraction and color segmentation in the HSV space. In this study, a simple background subtraction technique is used based on inter-frame difference. Foreground/background models are learnt and updated over time. The background model is initialized with the first frame of the video sequence. For the extraction of foreground objects, the current frame is subtracted from the background model and any significant difference is labeled as foreground. If no foreground object is identified, the current frame becomes the background model. On the saturation plane, the shaft of the probe is highlighted in dark grey on a bright background. Therefore, foreground pixels (Fig. 2 (a)) are used as seeds for region growing in the saturation color plane (Fig. 2 (b)) to segment the probe shaft as shown in Fig. 2(c).

In order to identify the tip of the probe, the centroid of the shaft is extracted. The tangentials of the shaft are detected at the global maxima of the Hough transform and the axis of the shaft is computed as the eigenvector corresponding to the smallest eigenvalue of the moment of inertia. The localization of the tip of the probe is performed with respect to a reference point located at the intersection of the shaft and the 

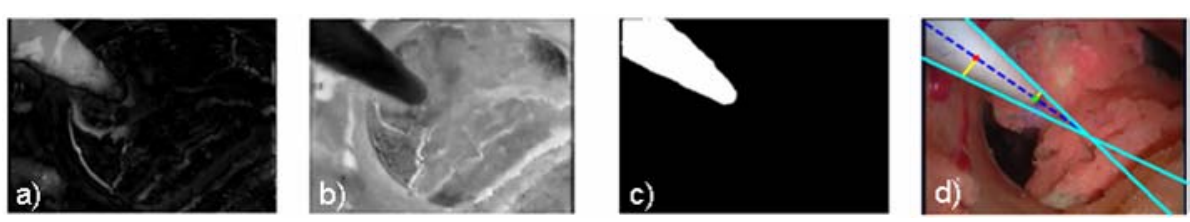

Fig. 2. Probe tracking and biopsy site estimation within the image plane; (a) background subtraction, (b) color saturation distribution within the image, (c) segmented tool regions, and (d) the model fitted tool (centroid -red dot, the reference point - green dot, vanishing tangential lines - cyan, radius at the center of mass and at the reference point - yellow).

distal tip. The 3D position of the tip is estimated using a semi-model based approach assuming rigidity and incorporating prior knowledge of the width of the probe and the relationship between the reference point and the tip. The position of the reference point and the orientation of the shaft are estimated in 3D and the prior model enables the localization of the tip of the probe $b^{c}$ relative to the camera.

\subsection{Global Biopsy Mapping}

Following the steps in Section 2.2, the position of biopsy site $b^{c}$ is estimated in the camera coordinate system. It is transformed into the world coordinate system using:

$$
b^{w}=C^{w} b^{c}+c^{w}
$$

where $b^{w}$ is the biopsy site in the world coordinate system, $C^{w}$ and $c^{w}$ are the orientation and position of the camera in the global SLAM coordinate system. Although the 3D position of the biopsy site is now defined, this position is never directly observed or measured again. There are two reasons for this; the actual site on the tissue is usually occluded by the probe when the biopsy is taken, and there may not be any salient features at or around the biopsy site to be tracked. In this case, 2D tracking would fail. However, the strength of the proposed probabilistic map is that the position of the biopsy site can be updated without directly measuring it. This is made possible by the co-variance matrix which models the uncertainty of all the biopsy positions. The $i^{\text {th }}$ biopsy site $b_{i}^{w}$ is inserted into the state vector and the co-variance matrix $P$ is updated. The co-variance matrix is updated with the partial derivatives $\partial b_{i} / \partial x_{v}$ of the biopsy site with respect to the camera position, as well as the measurement model $\partial b_{i} / \partial h_{i}$ and measurement noise $R$ as shown in Eq (2).

$$
b_{i}^{w}=\left(\begin{array}{c}
x \\
y \\
z
\end{array}\right), \quad P=\left[\begin{array}{ccc}
P_{x x} & P_{x y_{1}} & P_{x x} \frac{\partial b_{i}^{T}}{\partial x_{v}} \\
P_{y_{1} x} & P_{y_{1} y_{1}} & P_{y_{1} x} \frac{\partial b_{i}^{T}}{\partial x_{v}} \\
\frac{\partial b_{i}^{w}}{\partial x_{v}} P_{x x} & \frac{\partial b_{i}^{w}}{\partial x_{v}} P_{x y_{1}} & \frac{\partial b_{i}^{w}}{\partial x_{v}} P_{x x} \frac{\partial b_{i}^{w T}}{\partial x_{v}}+\frac{\partial b_{i}^{w}}{\partial h_{i}} R \frac{\partial b_{i i}^{w}{ }^{T}}{\partial h_{i}}
\end{array}\right]
$$

where $x$ is the position of the endoscope and $y_{i}$ is the $i^{\text {th }}$ feature in the map.

The position and uncertainty of the biopsy sites are correlated to the camera position and the rest of the features in the map. Fig. 3 illustrates this sequential map building demonstrating how the camera, features and the biopsy sites are correlated and 

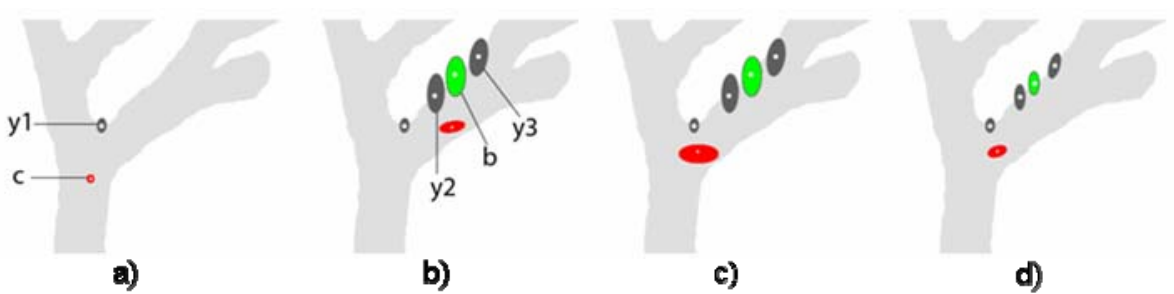

Fig. 3. (a-d) Schematic representation of sequential probabilistic mapping updates. The camera's position $\mathrm{c}$ is shown in red with the uncertainty represented by an ellipse, features $\mathrm{y} 1, \mathrm{y} 2$ and $y 3$ represented in dark gray, the biopsy site b shown in green and the tissue shown in light gray. (a) c measures y1 with low uncertainty, (b) $\mathrm{c}$ is navigated to a new position with growing uncertainty. Features y 2 and y3 are measured and biopsy b is taken. (c) c is navigated close to y1 and positional uncertainty increases. (d) Feature y1 is measured and the position estimate of $\mathrm{c}$ is improved. Resulting in an improved estimate of $\mathrm{b}$, as it is correlated to $\mathrm{c}$.

temporally updated. At the time when the biopsy site is observed, the uncertainty of the camera's position may be high, as illustrated in Fig. 3 (b) but the relative position of the biopsy site to surrounding features is well defined. Over time, the camera will re-measure these surrounding features in the map as in Fig. 3 (d) and the position estimation of the camera will improve, thus reducing the uncertainty. Therefore, the position estimation of the biopsy site will also improve as it is correlated to the position of the camera and will not drift away in the global map. To facilitate real-time examination, the biopsy sites $\left\{b_{1}^{w} \ldots b_{i}^{w}\right\}$ are visualized in this study by re-projecting the 3D points into the camera plane based on the intrinsic camera parameters and the estimated camera position from SLAM. This provides an augmented view of the biopsy sites for the operator.

\subsection{Experimental Set-Up}

The proposed approach has been validated on a silicon phantom of the airway coated with acrylic paint to provide realistic texture and internal reflections. Sponge cell structures were attached to the internal surface to enable optical biopsies to be taken using a confocal fluorescence endoscope system (Cellvizio, Mauna Kea Technologies, Paris). Validation was performed by measuring the accuracy of biopsy sites in the image space as the endoscope navigated through the phantom. The ground truth data used for comparison was collected using an optical tracking device (Northern Digital Inc, Ontario, Canada) and an experienced observer. To obtain the ground truth position of the camera, a rigid stereo laparoscope fitted with four optical markers was used. The position $c^{l}$ and orientation $C^{l}$ of the center of the left camera relative to the optical markers were acquired using standard hand-eye calibration [11]. This enabled the position of the camera to be calculated in the world coordinate system $c^{w}$ and $C^{w}$.To obtain the ground truth of the 3D biopsy site positions, the experienced observer manually identified the sites on the stereo images at the time when the biopsy was taken. By using the camera's intrinsic and extrinsic parameters, the 3D position $b^{c}$ of the biopsy site was obtained relative to the camera, and its position in 
the world coordinate system $b^{w}$ was determined as $b^{w}=C^{w *} b^{c}+c^{w}$. At each subsequent frame, the biopsy site $b^{w}$ was projected into the ground truth camera position $x=x_{o}-f k_{x}\left(b_{x}^{c} / b_{z}^{c}\right)$ and $y=y_{o}-f k_{y}\left(b_{y}^{c} / b_{z}^{c}\right)$ where $f k_{x}$ and $f k_{y}$ are the focal length and $x_{o}$ and $y_{o}$ are the principal point. To validate the proposed probe tracking approach, the probe was mounted in a rigid sheath. This evaluation step was combined with manually defined image coordinates of the probe's location.

\section{Results}

The proposed algorithm was validated on a two minute long stereo laparoscopic video sequence consisting of navigation to four different areas, including six biopsies and re-targeting previously taken biopsies.

Quantitative analysis of the position of the biopsy sites in the image plane is shown in Table 1. The average visual angle error for the position of the biopsy sites ranges from $1.18^{\circ}$ to $3.86^{\circ}$. Figs. 4 (d-e) show the estimated biopsy site position and ground truth position of site three over a short sequence before the site goes out of view. Accuracy of the biopsy position estimation is affected by the proximity of the camera to the site where close proximity leads to a magnification of the error. Fig. 5 illustrates the results of the augmented biopsy sites at different stages of the procedure where changes in illumination, scale and view point are experienced. Fig. 5 demonstrates the practical value and clinical relevance of the proposed method; the entire procedure is represented where six biopsies are taken and added to the global map, including the associated biopsy images of the sponge cell structures.

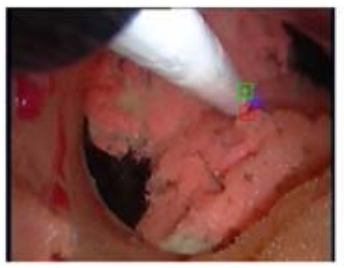

a)

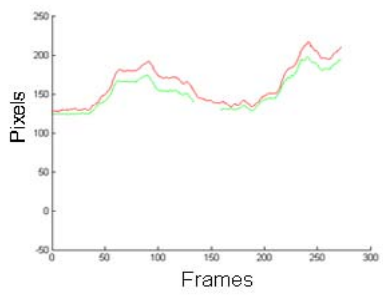

d)

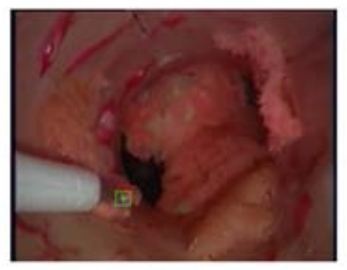

b)

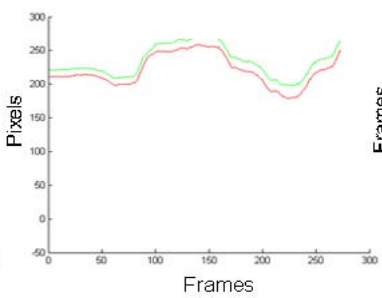

e)

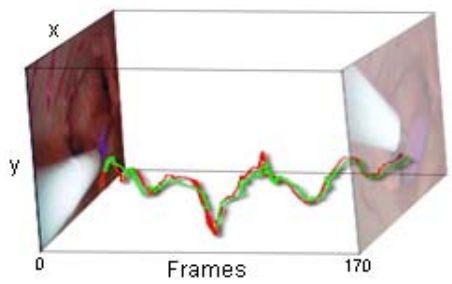

c)

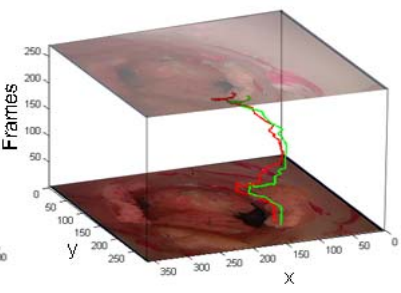

f)

Fig. 4. (a-c) Probe tracking: Ground truth (red) and estimated (green) position of probe at (a) site six and (b) site three. (c) Ground truth (red) and tracked probe position (green) during navigation between biopsy sites. (d-f) Augmented biopsy site three: (d-e) the X and Y projected pixel error showing the site being tracked (f) the ground truth projected position (red) and the estimated position (green) for a short section of the procedure. 
Table 1. Average error of biopsy site estimation and probe tracking for phantom experiments

\begin{tabular}{|c|c|c|c|c|}
\hline \multirow[b]{2}{*}{ Biopsy sites } & \multicolumn{2}{|c|}{ Probe tracking } & \multicolumn{2}{|c|}{ Augmented biopsy sites } \\
\hline & $\begin{array}{l}\text { Average visual } \\
\text { angle error }\end{array}$ & $\begin{array}{l}\text { Percent of } \\
\text { FOV }\end{array}$ & $\begin{array}{c}\text { Visual angle } \\
\text { error }\end{array}$ & $\begin{array}{l}\text { Percent of } \\
\text { FOV }\end{array}$ \\
\hline 1 & $1.85^{\circ}$ & $4.89 \%$ & $2.34^{\circ}$ & $5.37 \%$ \\
\hline 2 & $1.33^{\circ}$ & $3.59 \%$ & $3.06^{\circ}$ & $7.58 \%$ \\
\hline 3 & $0.87^{\circ}$ & $2.29 \%$ & $2.22^{\circ}$ & $5.59 \%$ \\
\hline 4 & $0.86^{\circ}$ & $2.23 \%$ & $1.18^{\circ}$ & $2.99 \%$ \\
\hline 5 & $0.81^{\circ}$ & $1.75 \%$ & $2.06^{\circ}$ & $4.61 \%$ \\
\hline 6 & $3.22^{\circ}$ & $8.88 \%$ & $3.86^{\circ}$ & $10.09 \%$ \\
\hline
\end{tabular}

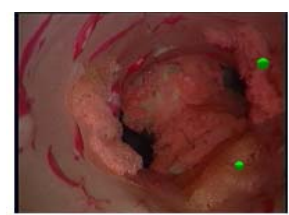

a)

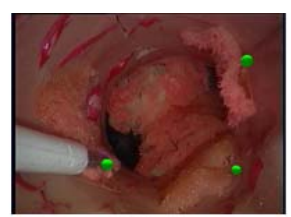

b)

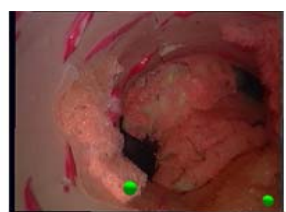

c)

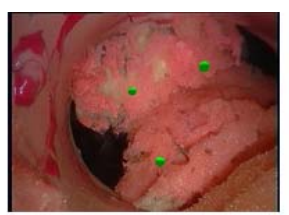

d)

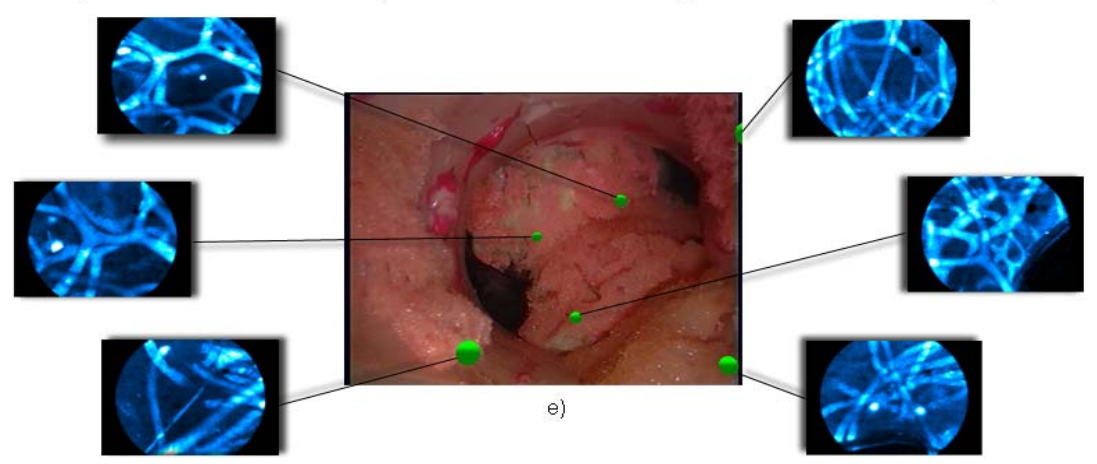

Fig. 5. (a-d) Biopsy site position (green spheres). The spheres are $2 \mathrm{~mm}$ in diameter and appear in different sizes when they are projected onto the image under perspective projection; (e) shows the six biopsy sites with corresponding micro-confocal fluorescence endoscope images.

Detailed quantitative analysis of the probe tracking when the biopsies are taken is shown in Table 1. The tracking errors range from $0.81^{\circ}$ to $3.22^{\circ}$ of the visual angle and an example error distribution is illustrated in Fig. 4 (a-b). Quantitative analysis of the probe tracking on the whole sequence gave an average visual angle error of $2.87^{\circ}$. The sensitivity and specificity were 0.9706 and 0.9892 , respectively. As expected, the accuracy deteriorates when the probe is introduced and removed from the scene as a part of the shaft is occluded, or when the probe is very close to the camera.

\section{Conclusion}

In this paper we have proposed a novel approach for microconfocal optical biopsy tracking which can be used to augment intra-operative navigation and retargeting of previously examined tissue regions. The system has been validated with a detailed 
phantom experiment and we have demonstrated that this approach can accurately project the location of biopsy sites, thus enabling its practical clinical use. The proposed method requires no prior information of the tissue geometry and can operate consistently in a sparse feature environment. The proposed method is robust to small local deformation and rigid global motion. Modeling large scale nonlinear tissue deformation, however, is not trivial and will be addressed in future work.

Acknowledgments. We gratefully acknowledge support from the EPSRC and the Technology Strategy Board grants DT/F003064/1 and DT/E011101/1.

\section{References}

1. Meining, A., Bajbouj, M., von Delius, S., Prinz, C.: Confocal Laser Scanning Microscopy for in vivo Histopathology of the Gastrointestinal Tract. Arab Journal of Gastroenterology $8,1-4(2007)$

2. Thiberville, L., Moreno-Swirc, S., Vercauteren, T., Peltier, E., Cavé, C., Bourg Heckly, G.: In Vivo Imaging of the Bronchial Wall Microstructure Using Fibered Confocal Fluorescence Microscopy. American Journal of Respiratory and Critical Care Medicine 175, 22 $31(2007)$

3. Van Dam, J.: Novel methods of enhanced endoscopic imaging. GUT 52(4) (2003)

4. Wengert, C., Cattin, P.C., Duff, J.M., Székely, G.: Markerless Endoscopic Registration and Referencing. In: Larsen, R., Nielsen, M., Sporring, J. (eds.) MICCAI 2006. LNCS, vol. 4190, pp. 816-823. Springer, Heidelberg (2006)

5. Wu, C.-H., Sun, Y.-N., Chen, Y.-C., Chang, C.-C.: Endoscopic Feature Tracking and Scale-Invariant Estimation of Soft-Tissue Structures. IEICE Transactions on Information and Systems 2, 351-360 (2008)

6. Atasoy, S., Noonan, D.P., Benhimane, S., Navab, N., Yang, G.-Z.: A global approach for automatic fibroscopic video mosaicing in minimally invasive diagnosis. In: Metaxas, D., Axel, L., Fichtinger, G., Székely, G. (eds.) MICCAI 2008, Part I. LNCS, vol. 5241, pp. 850-857. Springer, Heidelberg (2008)

7. Lerotic, M., Chung, A.J., Clark, J., Valibeik, S., Yang, G.-Z.: Dynamic View Expansion for Enhanced Navigation in Natural Orifice Transluminal Endoscopic Surgery. In: Metaxas, D., Axel, L., Fichtinger, G., Székely, G. (eds.) MICCAI 2008, Part II. LNCS, vol. 5242, pp. 467-475. Springer, Heidelberg (2008)

8. Wengert, C., Bossard, L., Häberling, A., Baur, C., Székely, G., Cattin, P.C.: Endoscopic Navigation for Minimally Invasive Suturing. In: Ayache, N., Ourselin, S., Maeder, A. (eds.) MICCAI 2007, Part II. LNCS, vol. 4792, pp. 620-627. Springer, Heidelberg (2007)

9. Krupa, A., Gangloff, J., Doignon, C., de Mathelin, M.F., Morel, G., Leroy, J., Soler, L., Marescaux, J.: Autonomous 3-D positioning of surgical instruments in robotized laparoscopic surgery using visual servoing. IEEE Transactions on Robotics and Automation 19, 842-853 (2003)

10. Mountney, P., Stoyanov, D., Davison, A.J., Yang, G.-Z.: Simultaneous Stereoscope Localization and Soft-Tissue Mapping for Minimal Invasive Surgery. In: Larsen, R., Nielsen, M., Sporring, J. (eds.) MICCAI 2006. LNCS, vol. 4190, pp. 347-354. Springer, Heidelberg (2007)

11. Tsai, R., Lenz, R.: Real Time Versatile Robotic Hand/Eye Calibration using 3D Machine Vision. In: Proc. ICRA 1988, pp. 554-561 (1988) 